\title{
Regularization and stability estimates for an inverse source problem of the radially symmetric parabolic equation
}

\section{Wei Cheng*}

"Correspondence:

chwei670815@163.com

College of Science, Henan

University of Technology,

Zhengzhou, 450001, P.R. China

\section{Springer}

\begin{abstract}
We consider an inverse problem of determining an unknown source term in the radially symmetric parabolic equation from a noisy final data and prove the uniqueness of solution for the problem. Using the Hölder inequality, we obtain a conditional stability for the space-dependent source term. A modified quasi-reversibility method is applied to deal with the ill-posedness of the problem. A Hölder-type error estimate between the approximate solution and the exact solution is provided by introducing some technical inequalities and choosing a suitable regularization parameter.
\end{abstract}

MSC: 35R25; 35R30; 65J20; 65M30

Keywords: ill-posed problem; inverse source problem; parabolic equation; quasi-reversibility method; error estimate

\section{Introduction}

Inverse source problems occur in many branches of engineering sciences, for example, heat conduction, reaction diffusion, pollutant detection, crack identification, geophysical prospecting and electromagnetic theory. These problems are typically ill-posed in the sense of Hadamard [1]. In other words, the solution (if it exists) does not depend continuously on measured data. So, the numerical simulation is very difficult and some special regularization methods and stability estimates are required.

The inverse source problems have been investigated in many papers; for example, the existence and uniqueness of the solution were investigated in [2, 3], the conditional stability and the data compatibility were studied in [4-10], and the numerical algorithms for the identification problem can be found in [11-17]. In [18], Yang and Fu solved an inverse problem for determining a heat source in a parabolic equation by a mollification regularization method, and they gave two kinds of explicit error estimates by using an a priori and an a posteriori regularization parameter choice rule, respectively. In [19], Wei and Wang used a modified quasi-boundary value method to deal with an inverse source problem of the time-fractional diffusion equation and provided two kinds of convergence rates. Cheng et $a l$. in [20] solved the identification of an unknown source term in radial heat conduction by a spectral method and gave a logarithmic-type error estimate. Yang et al. in [21] applied a quasi-boundary value regularization method for identifying an unknown source

(c) 2015 Cheng: licensee Springer. This is an Open Access article distributed under the terms of the Creative Commons Attribution License (http://creativecommons.org/licenses/by/4.0), which permits unrestricted use, distribution, and reproduction in any medium, provided the original work is properly credited. 
in the Poisson equation. However, to our knowledge, the research into the inverse source identification problem is mainly devoted to numerical methods. The stability theory with explicit error estimate for the problem is still limited.

In this paper, we apply a modified quasi-reversibility method to deal with an inverse source identification problem of a radially symmetric parabolic equation and obtain a Hölder-type error estimate between the approximate solution and the exact solution. The physical model we considered is a sphere of radius $r_{0}$ with initial state, and it is considered radially symmetric and with the surface state distribution function remaining zero. The correspondingly mathematical model is the following radially symmetric parabolic equation:

$$
\frac{\partial u}{\partial t}-\frac{\partial^{2} u}{\partial r^{2}}-\frac{2}{r} \frac{\partial u}{\partial r}=f(r), \quad 0<r<r_{0}, 0<t<T,
$$

with the boundary conditions

$$
u\left(r_{0}, t\right)=0, \quad \lim _{r \rightarrow 0} u(r, t) \text { bounded, } \quad 0 \leq t \leq T,
$$

and the initial condition and final observation at $t=T$,

$$
u(r, 0)=0, \quad u(r, T)=g(r), \quad 0 \leq r \leq r_{0},
$$

where $r$ denotes the radial coordinate, $u$ represents state function, and $f$ is physical laws, which means source term here. The inverse source identification problem (1.1)-(1.3) is to determine the unknown source term $f(r)$ from the noisy final data $g(r)$.

The quasi-reversibility method was first proposed by Lattès and Lions in [22]. This method consists in replacing the former second-order ill-posed problem into a family of well-posed fourth-order problems that depend on a regularization parameter $\alpha$. The solution of quasi-reversibility is close to the exact solution when $\alpha$ is small. This method has been used to solve various types of inverse problems such as inverse heat conduction problem [23, 24], backward heat conduction problem [25], the Cauchy problem of Laplace equation [26, 27] and Cauchy problem for the modified Helmholtz equation [28].

In this study, we propose a modified version of quasi-reversibility method to solve the inverse source problem (1.1)-(1.3), i.e., adding a perturbation term in the parabolic equation (1.1) to form an approximate problem:

$$
\left\{\begin{array}{lll}
\frac{\partial u}{\partial t}-\frac{\partial^{2} u}{\partial r^{2}}-\frac{2}{r} \frac{\partial u}{\partial r}=f(r)-\alpha \frac{r_{0}^{2}}{\pi^{2} r}(r f(r))^{\prime \prime}, & 0<r<r_{0}, 0<t<T, \\
u(r, 0)=0, & u(r, T)=g^{\delta}(r), & 0 \leq r \leq r_{0}, \\
u\left(r_{0}, t\right)=0, & \lim _{r \rightarrow 0} u(r, t) \text { bounded, } & 0 \leq t \leq T,
\end{array}\right.
$$

where $\alpha$ plays a role of regularization parameter, data $g^{\delta}$ represents the measured data of function $g$. For this modification, we can obtain a Hölder-type error estimate with an a priori choice of regularization parameter.

The paper is organized as follows. In Section 2, the ill-posedness of problem (1.1)-(1.3) is described. In Section 3, we prove the uniqueness of solution and obtain a conditional stability for the inverse source problem (1.1)-(1.3) by using the Hölder inequality. Introducing some technical inequalities and choosing a suitable regularization parameter, in 
Section 4 we present a Hölder-type error estimate between the approximate solution and the exact solution for the modified quasi-reversibility method.

\section{III-posedness of problem (1.1)-(1.3)}

In this section, we derive an analytical solution for the inverse source problem by the eigenfunction expansion and analyze the ill-posedness of inverse source problem (1.1)-(1.3). Throughout this paper, we denote by $L^{2}\left[0, r_{0} ; r^{2}\right]$ the Hilbert space of Lebesgue measurable functions $h$ with weight $r^{2}$ on $\left[0, r_{0}\right]$, and $(\cdot, \cdot)$ and $\|\cdot\|$ denote the inner product and the norm on $L^{2}\left[0, r_{0} ; r^{2}\right]$, respectively, with the norm

$$
\|h\|=\left(\int_{0}^{r_{0}} r^{2}|h(r)|^{2} d r\right)^{\frac{1}{2}}
$$

If the solution of problem (1.1)-(1.3) exists, then it must be unique, which will be given in Section 3.

Applying separation of variables, we can obtain the eigenvalues and corresponding eigenfunctions of problem (1.1)-(1.3):

$$
\lambda_{n}=\left(\frac{n \pi}{r_{0}}\right)^{2} \quad \text { and } \quad R_{n}(r)=j_{0}\left(\frac{n \pi r}{r_{0}}\right), \quad n=1,2, \ldots
$$

where $j_{0}(x)=\frac{\sin x}{x}$ denotes a spherical Bessel function of the first kind [29]. Then, using the eigenfunction method, we suppose that the solution $u(r, t)$ and nonhomogeneous term $f(r)$ of problem (1.1)-(1.3) can be represented as follows:

$$
\begin{aligned}
& u(r, t)=\sum_{n=1}^{\infty} u_{n}(t) j_{0}\left(\frac{n \pi r}{r_{0}}\right), \\
& f(r)=\sum_{n=1}^{\infty} f_{n} j_{0}\left(\frac{n \pi r}{r_{0}}\right) .
\end{aligned}
$$

Applying the properties of $j_{0}(x)$, the eigenfunctions system $j_{0}\left(\frac{n \pi r}{r_{0}}\right)$ is complete and orthogonal with weight $r^{2}$ on $\left[0, r_{0}\right]$. Substituting (2.1) and (2.2) into equation (1.1) and the initial condition in (1.2), we have that $u_{n}(t)$ satisfies

$$
\left\{\begin{array}{l}
u_{n}^{\prime}(t)+\left(n \pi / r_{0}\right)^{2} u_{n}(t)=f_{n} \\
u_{n}(0)=0
\end{array}\right.
$$

Solving this initial problem yields

$$
u_{n}(t)=\int_{0}^{t} f_{n} e^{-\left(\frac{n \pi}{r_{0}}\right)^{2}(t-\tau)} d \tau, \quad n=1,2, \ldots
$$

Combining (2.1) with (2.4), we have

$$
u(r, t)=\sum_{n=1}^{\infty} f_{n}\left(\int_{0}^{t} e^{-\left(\frac{n \pi}{r_{0}}\right)^{2}(t-\tau)} d \tau\right) j_{0}\left(\frac{n \pi r}{r_{0}}\right) .
$$


From (1.2) at $t=T$,

$$
g(r)=\sum_{n=1}^{\infty} f_{n}\left(\int_{0}^{T} e^{-\left(\frac{n \pi}{r_{0}}\right)^{2}(T-\tau)} d \tau\right) j_{0}\left(\frac{n \pi r}{r_{0}}\right) .
$$

Thus, there holds

$$
f_{n}=\frac{2\left((n \pi)^{2} / r_{0}^{3}\right)}{\int_{0}^{T} e^{-\left(\frac{n \pi}{r_{0}}\right)^{2}(T-\tau)} d \tau} \int_{0}^{r_{0}} r^{2} g(r) j_{0}\left(\frac{n \pi r}{r_{0}}\right) d r, \quad n=1,2, \ldots
$$

since

$$
\int_{0}^{T} e^{-\left(\frac{n \pi}{r_{0}}\right)^{2}(T-\tau)} d \tau=\left(\frac{r_{0}}{n \pi}\right)^{2}\left(1-e^{-\left(\frac{n \pi}{r_{0}}\right)^{2} T}\right)
$$

then formula (2.5) becomes

$$
g(r)=\sum_{n=1}^{\infty} f_{n}\left(\frac{r_{0}}{n \pi}\right)^{2}\left(1-e^{-\left(\frac{n \pi}{r_{0}}\right)^{2} T}\right) j_{0}\left(\frac{n \pi r}{r_{0}}\right) .
$$

Define

$$
\varphi_{n}(r)=\frac{\sqrt{2} n \pi}{\sqrt{r_{0}^{3}}} j_{0}\left(\frac{n \pi r}{r_{0}}\right),
$$

this eigenfunctions system is orthonormal with weight $r^{2}$ on $\left[0, r_{0}\right]$ and a complete system in $L^{2}\left[0, r_{0} ; r^{2}\right]$. Combining (2.6) with (2.8), formula (2.7) can be rewritten as

$$
g(r)=\sum_{n=1}^{\infty}\left(\frac{r_{0}}{n \pi}\right)^{2}\left(1-e^{-\left(\frac{n \pi}{r_{0}}\right)^{2} T}\right)\left(f, \varphi_{n}\right) \varphi_{n}(r)
$$

In practical applications, the input data $g$ can only be measured, so we actually have the measured data function $g^{\delta}(\cdot)$ which belongs to $L^{2}\left[0, r_{0} ; r^{2}\right]$ and satisfies

$$
\left\|g-g^{\delta}\right\| \leq \delta
$$

where $\delta>0$ denotes the noise level.

We introduce an operator $K: f(\cdot) \rightarrow g(\cdot)$, then we have

$$
g(r)=K f(r)=\sum_{n=1}^{\infty}\left(\frac{r_{0}}{n \pi}\right)^{2}\left(1-e^{-\left(\frac{n \pi}{r_{0}}\right)^{2} T}\right)\left(f, \varphi_{n}\right) \varphi_{n} .
$$

It is easy to see that $K$ is linear self-adjoint compact operator with eigenvalues

$$
k_{n}=\left(\frac{r_{0}}{n \pi}\right)^{2}\left(1-e^{-\left(\frac{n \pi}{r_{0}}\right)^{2} T}\right)
$$

and eigenelements $\varphi_{n}$. From formula (2.11), we have

$$
\left(g, \varphi_{n}\right)=\left(f, \varphi_{n}\right) k_{n}
$$


then

$$
f(r)=\sum_{n=1}^{\infty}\left(f, \varphi_{n}\right) \varphi_{n}=\sum_{n=1}^{\infty} k_{n}^{-1}\left(g, \varphi_{n}\right) \varphi_{n}
$$

Since the eigenvalues $k_{n}$ of the operator $K$ decay, we realize that problem (1.1)-(1.3) is an ill-posed problem.

It is well known that for any ill-posed problems an a priori bound assumption for the exact solution is needed and necessary. Otherwise, the convergence of the regularized approximation solution will not be obtained or the convergence rate can be arbitrarily slow [30]. Assume also that there exists an a priori condition for problem (1.1)-(1.3):

$$
\|f\|_{p} \leq E, \quad p>0
$$

where $\|f\|_{p}$ is defined by

$$
\|f\|_{p}=\left(\sum_{n=1}^{\infty}\left(1+n^{2}\right)^{p}\left|\left(f, \varphi_{n}\right)\right|^{2}\right)^{\frac{1}{2}}
$$

\section{Uniqueness and conditional stability for problem (1.1)-(1.3)}

In this section, we provide the uniqueness and conditional stability in Theorems 3.1 and 3.2 , respectively.

Let $g$ be a known function in $L^{2}\left[0, r_{0} ; r^{2}\right]$. We consider the problem of finding a pair of functions $(u(r, t), f(r))$.

Theorem 3.1 If $u_{i} \in C^{2,1}\left(\left(0, r_{0}\right) \times(0, T]\right), f_{i} \in L^{2}\left[0, r_{0} ; r^{2}\right](i=1,2)$. Let $\left(u_{i}, f_{i}\right)(i=1,2)$ satisfy problem (1.1)-(1.3), then $\left(u_{1}, f_{1}\right)=\left(u_{2}, f_{2}\right)$.

Proof Put $\tilde{u}=u_{1}-u_{2}, \tilde{f}=f_{1}-f_{2}$, it is easy to know that $\tilde{u}$ satisfies

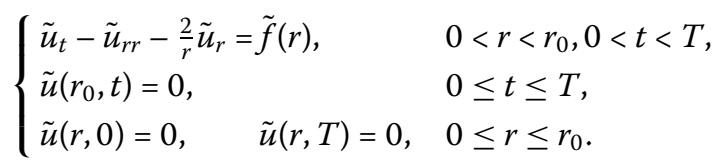

We now introduce a function

$$
v(r, t)=\tilde{u}(r, t)+F(r),
$$

where

$$
F(r)=\int_{0}^{r} \xi^{-2} \int_{0}^{\xi} \tau^{2} \tilde{f}(\tau) d \tau d \xi-\int_{0}^{r_{0}} \xi^{-2} \int_{0}^{\xi} \tau^{2} \tilde{f}(\tau) d \tau d \xi .
$$

According to formulas (3.1)-(3.3), we can obtain that the function $v(r, t)$ satisfies

$$
\begin{aligned}
& v_{t}-v_{r r}-\frac{2}{r} v_{r}=0, \quad 0<r<r_{0}, 0<t<T, \\
& v\left(r_{0}, t\right)=0, \quad 0 \leq t \leq T,
\end{aligned}
$$




$$
v(r, 0)=v(r, T)=F(r), \quad 0 \leq r \leq r_{0} .
$$

From (3.4)-(3.6), we have

$$
\begin{aligned}
\int_{0}^{T} \int_{0}^{r_{0}} r^{2} v\left(v_{t}-v_{r r}-\frac{2}{r} v_{r}\right) d r d t \\
=\left.\frac{1}{2} \int_{0}^{r_{0}} r^{2} v^{2}\right|_{t=0} ^{t=T} d r-\int_{0}^{T} \int_{0}^{r_{0}} v d\left(r^{2} v_{r}\right) d t \\
=\frac{1}{2} \int_{0}^{r_{0}} r^{2}\left(v^{2}(r, T)-v^{2}(r, 0)\right) d r \\
\quad-\int_{0}^{T}\left(\left.r^{2} v v_{r}\right|_{r=0} ^{r=r_{0}}-\int_{0}^{r_{0}} r^{2} v_{r}^{2} d r\right) d t \\
=\int_{0}^{T} \int_{0}^{r_{0}} r^{2} v_{r}^{2} d r d t=0 .
\end{aligned}
$$

Thus,

$$
v_{r}^{2}=0
$$

i.e.,

$$
v(r, t)=C(t), \quad 0 \leq r \leq r_{0} .
$$

So there holds $v\left(r_{0}, t\right)=C(t)$. Combining this with (3.5) yields

$$
v(r, t) \equiv 0, \quad(r, t) \in\left[0, r_{0}\right] \times[0, T]
$$

From (3.7) and (3.6), we get

$$
F(r) \equiv 0, \quad(r, t) \in\left[0, r_{0}\right] \times[0, T]
$$

and

$$
\int_{0}^{r} \xi^{-2} \int_{0}^{\xi} \tau^{2} \tilde{f}(\tau) d \tau d \xi-\int_{0}^{r_{0}} \xi^{-2} \int_{0}^{\xi} \tau^{2} \tilde{f}(\tau) d \tau d \xi=0 .
$$

Differentiating (3.9) with respect to $r$, we obtain $\tilde{f} \equiv 0$. Substituting (3.7) and (3.8) into (3.2) yields $\tilde{u} \equiv 0$. The uniqueness of inverse source problem (1.1)-(1.3) is proved.

We give a conditional stability for the inverse source problem (1.1)-(1.3) in the following theorem.

Theorem 3.2 Let $f(r)$ be the solution of the inverse source problem (1.1)-(1.3), and condition (2.14) be satisfied, then the following estimate holds:

$$
\|f\| \leq\left(\frac{e^{\left(\pi / r_{0}\right)^{2} T} \pi^{2}}{\left(e^{\left(\pi / r_{0}\right)^{2} T}-1\right) r_{0}^{2}}\right)^{\frac{p}{p+2}} E^{\frac{2}{p+2}}\|g\|^{\frac{p}{p+2}} .
$$


Proof From (2.13) and the Hölder inequality, the following holds:

$$
\begin{aligned}
& \|f\|^{2}=\left\|\sum_{n=1}^{\infty} k_{n}^{-1}\left(g, \varphi_{n}\right) \varphi_{n}\right\|^{2}=\sum_{n=1}^{\infty}\left|k_{n}\right|^{-2}\left|g_{n}\right|^{2}=\sum_{n=1}^{\infty}\left|k_{n}\right|^{-2}\left|g_{n}\right|^{\frac{4}{p+2}}\left|g_{n}\right|^{\frac{2 p}{p+2}} \\
& \leq\left[\sum_{n=1}^{\infty}\left(\left|k_{n}\right|^{-2}\left|g_{n}\right|^{\frac{4}{p+2}}\right)^{\frac{p+2}{2}}\right]^{\frac{2}{p+2}}\left[\sum_{n=1}^{\infty}\left(\left|g_{n}\right|^{\frac{2 p}{p+2}}\right)^{\frac{p+2}{p}}\right]^{\frac{p}{p+2}} \\
& =\left[\sum_{n=1}^{\infty}\left|k_{n}^{-1}\right|^{p+2}\left|g_{n}\right|^{2}\right]^{\frac{2}{p+2}}\left[\sum_{n=1}^{\infty}\left|g_{n}\right|^{2}\right]^{\frac{p}{p+2}} \\
& =\left[\sum_{n=1}^{\infty}\left|k_{n}^{-1}\right|^{p}\left|k_{n}^{-1} g_{n}\right|^{2}\right]^{\frac{2}{p+2}}\|g\|^{\frac{2 p}{p+2}} \\
& =\left[\sum_{n=1}^{\infty}\left|\frac{\left(n \pi / r_{0}\right)^{2}}{1-e^{-\left(n \pi / r_{0}\right)^{2} T}}\right|^{p}\left(1+n^{2}\right)^{-p}\left(1+n^{2}\right)^{p}\left|f_{n}\right|^{2}\right]^{\frac{2}{p+2}}\|g\|^{\frac{2 p}{p+2}} \\
& \leq \max _{n \in \mathbb{N}}\left|\left(\frac{\pi}{r_{0}}\right)^{2} \frac{e^{\left(n \pi / r_{0}\right)^{2} T}}{e^{\left(n \pi / r_{0}\right)^{2} T}-1}\right|^{\frac{2 p}{p+2}}\left[\sum_{n=1}^{\infty}\left(1+n^{2}\right)^{p}\left|f_{n}\right|^{2}\right]^{\frac{2}{p+2}}\|g\|^{\frac{2 p}{p+2}} \\
& \leq\left(\frac{e^{\left(\pi / r_{0}\right)^{2} T} \pi^{2}}{\left(e^{\left(\pi / r_{0}\right)^{2} T}-1\right) r_{0}^{2}}\right)^{\frac{2 p}{p+2}} E^{\frac{4}{p+2}}\|g\|^{\frac{2 p}{p+2}} .
\end{aligned}
$$

The proof is completed.

\section{Modified quasi-reversibility method and error estimate}

In this section, we propose a modified quasi-reversibility method to solve problem (1.1)(1.3) and give a Hölder-type error estimate with some technical inequalities and an a priori regularization parameter choice rule.

Let $\left(u_{\alpha}^{\delta}(r, t), f_{\alpha}^{\delta}(r)\right)$ be the solution of the following regularized problem:

$$
\begin{cases}\frac{\partial u_{\alpha}^{\delta}}{\partial t}-\frac{\partial^{2} u_{\alpha}^{\delta}}{\partial r^{2}}-\frac{2}{r} \frac{\partial u_{\alpha}^{\delta}}{\partial r}=f_{\alpha}^{\delta}(r)-\alpha \frac{r_{0}^{2}}{\pi^{2} r}\left(r f_{\alpha}^{\delta}(r)\right)^{\prime \prime}, & 0<r<r_{0}, 0<t<T, \\ u_{\alpha}^{\delta}(r, 0)=0, \quad u_{\alpha}^{\delta}(r, T)=g^{\delta}(r), & 0 \leq r \leq r_{0}, \\ u_{\alpha}^{\delta}\left(r_{0}, t\right)=0, \quad \lim _{r \rightarrow 0} u_{\alpha}^{\delta}(r, t) \text { bounded, } & 0 \leq t \leq T,\end{cases}
$$

where $\alpha>0$ is a regularization parameter.

Similar to the derivation process of formula (2.13), we can obtain the solution of problem (4.1) as follows:

$$
f_{\alpha}^{\delta}(r)=\sum_{n=1}^{\infty} \frac{k_{n}^{-1}}{1+\alpha n^{2}}\left(g^{\delta}, \varphi_{n}\right) \varphi_{n}
$$

We call $f_{\alpha}^{\delta}(r)$ above the quasi-reversibility approximations of the solution $f(r)$ of problem (1.1)-(1.3).

Before giving an error estimate, we present firstly the following lemma which is crucial for error estimate. 
Lemma 4.1 Let $x \geq 1$, then we have the inequality

$$
\frac{1}{1-e^{-x}} \leq \frac{e}{e-1} .
$$

Lemma 4.2 If constants $\alpha>0, p>0$, then we obtain the inequality

$$
\max _{n \in \mathbb{N}} \frac{\alpha n^{2}}{1+\alpha n^{2}}\left(1+n^{2}\right)^{-\frac{p}{2}} \leq \begin{cases}\alpha^{\frac{p}{2}}, & 0<p<2 \\ \alpha, & p \geq 2\end{cases}
$$

Proof Let

$$
\psi(n)=\frac{\alpha n^{2}}{1+\alpha n^{2}}\left(1+n^{2}\right)^{-\frac{p}{2}} .
$$

The proof of (4.4) is divided into two cases.

Case I. For large values of $n$, i.e., for $n \geq n_{0}:=\alpha^{-\frac{1}{2}}$, we have

$$
\psi(n) \leq\left(1+n^{2}\right)^{-\frac{p}{2}} \leq n_{0}^{-p}=\alpha^{\frac{p}{2}} .
$$

Case II. For $n<n_{0}$, there holds

$$
\psi(n) \leq \alpha n^{2}\left(1+n^{2}\right)^{-\frac{p}{2}} .
$$

If $0<p<2$, inequality (4.7) becomes

$$
\psi(n) \leq \alpha n^{2} \cdot n^{-p} \leq \alpha^{\frac{p}{2}} .
$$

If $p \geq 2$, we obtain

$$
\psi(n) \leq \frac{\alpha n^{2}}{1+n^{2}} \leq \alpha
$$

According to (4.5)-(4.9), the proof of (4.4) is completed.

Theorem 4.3 Let $f(r)$ given by (2.13) be the exact source history for $r \in\left[0, r_{0}\right]$ and $f_{\alpha}^{\delta}(r)$ given by (4.2) be the regularized approximation source to $f(r)$. Suppose that the a priori condition (2.14) and the noise assumption (2.10) hold, then:

(1) If $0<p<2$ and select the regularization parameter $\alpha=\left(\frac{\delta}{E}\right)^{\frac{2}{p+2}}$, there holds the stability estimate

$$
\left\|f(\cdot)-f_{\alpha}^{\delta}(\cdot)\right\| \leq\left(1+\frac{\pi^{2} e^{\left(\pi / r_{0}\right)^{2} T}}{r_{0}^{2}\left(e^{\left(\pi / r_{0}\right)^{2} T}-1\right)}\right) E^{\frac{2}{p+2}} \delta^{\frac{p}{p+2}}
$$

(2) If $p \geq 2$ and choose the regularization parameter $\alpha=\left(\frac{\delta}{E}\right)^{\frac{1}{2}}$, there holds the stability estimate

$$
\left\|f(\cdot)-f_{\alpha}^{\delta}(\cdot)\right\| \leq\left(1+\frac{\pi^{2} e^{\left(\pi / r_{0}\right)^{2} T}}{r_{0}^{2}\left(e^{\left(\pi / r_{0}\right)^{2} T}-1\right)}\right) E^{\frac{1}{2}} \delta^{\frac{1}{2}}
$$


Proof Due to (2.13) and (4.2), there holds

$$
\begin{aligned}
& \left\|f(\cdot)-f_{\alpha}^{\delta}(\cdot)\right\|=\left\|\sum_{n=1}^{\infty} k_{n}^{-1}\left(g, \varphi_{n}\right) \varphi_{n}-\sum_{n=1}^{\infty} \frac{k_{n}^{-1}}{1+\alpha n^{2}}\left(g^{\delta}, \varphi_{n}\right) \varphi_{n}\right\| \\
& \leq\left\|\sum_{n=1}^{\infty} k_{n}^{-1}\left(g, \varphi_{n}\right) \varphi_{n}-\sum_{n=1}^{\infty} \frac{k_{n}^{-1}}{1+\alpha n^{2}}\left(g, \varphi_{n}\right) \varphi_{n}\right\| \\
& +\left\|\sum_{n=1}^{\infty} \frac{k_{n}^{-1}}{1+\alpha n^{2}}\left(g-g^{\delta}, \varphi_{n}\right) \varphi_{n}\right\| \\
& \leq \sup _{n \in \mathbb{N}} \frac{\alpha n^{2}}{1+\alpha n^{2}}\left(1+n^{2}\right)^{-p / 2}\left\|\sum_{n=1}^{\infty}\left(1+n^{2}\right)^{p / 2} k_{n}^{-1}\left(g, \varphi_{n}\right) \varphi_{n}\right\|
\end{aligned}
$$

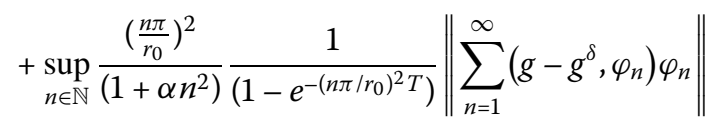

$$
\begin{aligned}
& \leq \sup _{n \in \mathbb{N}}\left(\frac{\pi}{r_{0}}\right)^{2} \frac{1}{\alpha} \frac{1}{\left(1-e^{\left.-\left(n \pi / r_{0}\right)^{2} T\right)}\right.}\left\|g-g_{\delta}\right\| \\
& +\sup _{n \in \mathbb{N}} \frac{\alpha n^{2}}{1+\alpha n^{2}}\left(1+n^{2}\right)^{-p / 2}\|f\|_{p} \text {. }
\end{aligned}
$$

Combining with conditions (2.10), (2.14) and inequalities (4.3), (4.4), we obtain

$$
\left\|f(\cdot)-f_{\alpha}^{\delta}(\cdot)\right\| \leq \frac{\pi^{2} e^{\left(\pi / r_{0}\right)^{2} T}}{r_{0}^{2}\left(e^{\left(\pi / r_{0}\right)^{2} T}-1\right)} \frac{\delta}{\alpha}+ \begin{cases}E \alpha^{\frac{p}{2}}, & 0<p<2 \\ E \alpha, & p \geq 2\end{cases}
$$

Choose the regularization parameter $\alpha$ by

$$
\alpha= \begin{cases}\left(\frac{\delta}{E}\right)^{\frac{2}{p+2}}, & 0<p<2 \\ \left(\frac{\delta}{E}\right)^{\frac{1}{2}}, & p \geq 2\end{cases}
$$

Thus, we have

$$
\left\|f(\cdot)-f_{\alpha}^{\delta}(\cdot)\right\| \leq \begin{cases}\left(1+\frac{\pi^{2} e^{\left(\pi / r_{0}\right)^{2} T}}{r_{0}^{2}\left(e^{\left(\pi / r_{0}\right)^{2} T}-1\right)}\right) E^{\frac{2}{p+2}} \delta^{\frac{p}{p+2}}, & 0<p<2, \\ \left(1+\frac{\pi^{2} e^{\left(\pi / r_{0}\right)^{2} T}}{r_{0}^{2}\left(e^{\left(\pi / r_{0}\right)^{2} T}-1\right)}\right) E^{\frac{1}{2}} \delta^{\frac{1}{2}}, & p \geq 2 .\end{cases}
$$

The proof is completed.

Remark 4.4 In general, the a priori bound $E$ is unknown in practice. In this case, for Theorem 4.3, with

$$
\alpha= \begin{cases}\delta^{\frac{2}{p+2}}, & 0<p<2 \\ \delta^{\frac{1}{2}}, & p \geq 2\end{cases}
$$

the following stability estimates hold:

$$
\left\|f(\cdot)-f_{\alpha}^{\delta}(\cdot)\right\| \leq \begin{cases}\left(E+\frac{\pi^{2} e^{\left(\pi / r_{0}\right)^{2} T}}{r_{0}^{2}\left(e^{\left(\pi / r_{0}\right)^{2} T}-1\right)}\right) \delta^{\frac{p}{p+2}}, & 0<p<2, \\ \left(E+\frac{\pi^{2} e^{\left(\pi / r_{0}\right)^{2} T}}{r_{0}^{2}\left(e^{\left(\pi / r_{0}\right)^{2} T}-1\right)}\right) \delta^{\frac{1}{2}}, & p \geq 2,\end{cases}
$$


where $E$ is only a bounded positive constant and it is not necessary to know it exactly.

\section{Competing interests}

The author declares that she has no competing interests.

\section{Acknowledgements}

The author would like to express her cordial thanks to the referees for their valuable comments and suggestions. This work was supported by the National Natural Science Foundation of China (No. 11171136), the NSF of Henan Province of China (Nos. 132300410231; 132300410013) and the Plan of Nature Science Fundamental Research in Henan University of Technology (No. 11JCYJ16).

\section{Received: 9 November 2014 Accepted: 6 April 2015 Published online: 17 April 2015}

\section{References}

1. Adams, RA: Sobolev Spaces. Pure and Applied Mathematics. Academic Press, New York (1975)

2. Savateev, EG: On problems of determining the source function in a parabolic equation. J. Inverse III-Posed Probl. 3(1), 83-102 (1995)

3. Solov'ev, W: Solvability of the inverse problem of finding a source, using overdetermination on the upper base for a parabolic equation. Differ. Equ. 25, 1114-1119(1990)

4. Cannon, JR, Pérez, ES: An inverse problem for the heat equation. Inverse Probl. 2(4), 395-403 (1986)

5. Cannon, JR, Duchateau, P: Structural identification of an unknown source term in a heat equation. Inverse Probl. 14, 535-551 (1998)

6. Yamamoto, M: Conditional stability in determination of force terms of heat equations in a rectangle. Math. Comput. Model. 18(1), 79-88 (1993)

7. Li, GS, Yamamoto, M: Stability analysis for determining a source term in a 1-D advection-dispersion equation. J. Inverse III-Posed Probl. 14(2), 147-155 (2006)

8. Choulli, M, Yamamoto, M: Conditional stability in determining a heat source. J. Inverse III-Posed Probl. 12(3), 233-243 (2004)

9. El Badia, A, Ha-Duong, T: On an inverse source problem for the heat equation. Application to a pollution detection problem. J. Inverse III-Posed Probl. 10(6), 585-600 (2002)

10. Li, GS: Data compatibility and conditional stability for an inverse source problem in the heat equation. Appl. Math. Comput. 173(1), 566-581 (2006)

11. Burykin, AA, Denisov, AM: Determination of the unknown sources in the heat-conduction equation. Comput. Math. Model. 8(4), 309-313 (1997)

12. Johansson, T, Lesnic, D: Determination of a spacewise dependent heat source. J. Comput. Appl. Math. 209(1), 66-80 (2007)

13. Yan, L, Yang, FL, Fu, CL: A meshless method for solving an inverse spacewise-dependent heat source problem. J. Comput. Phys. 228(1), 123-136 (2009)

14. Yan, L, Fu, CL, Dou, FF: A computational method for identifying a spacewise-dependent heat source. Int. J. Numer. Methods Biomed. Eng. 26, 597-608 (2010)

15. Shidfar, A, Babaei, A, Molabahrami, A: Solving the inverse problem of identifying an unknown source term in a parabolic equation. Comput. Math. Appl. 60, 1209-1213 (2010)

16. $\mathrm{Ma}, \mathrm{YJ}, \mathrm{Fu}, \mathrm{CL}$, Zhang, YX: Identification of an unknown source depending on both time and space variables by a variational method. Appl. Math. Model. 26(10), 1209-1213 (2012)

17. Wang, ZW, Liu, JJ: Identification of the pollution source from one-dimensional parabolic equation. Appl. Math. Comput. 219(8), 3400-3413 (2012)

18. Yang, F, Fu, CL: A mollification regularization for the spatial-dependent heat source problem. J. Comput. Appl. Math 255(1), 555-567 (2014)

19. Wei, T, Wang, JG: A modified quasi-boundary value method for an inverse source problem of the time-fractional diffusion equation. Appl. Numer. Math. 78, 95-111 (2014)

20. Cheng, W, Ma, YJ, Fu, CL: Identifying an unknown source term in radial heat conduction. Inverse Probl. Sci. Eng. 20(3), 335-349 (2012)

21. Yang, F, Zhang, M, Li, XX: A quasi-boundary value regularization method for identifying an unknown source in the Poisson equation. J. Inequal. Appl. 2014, 117 (2014)

22. Lattès, R, Lions, JL: The Method of Quasi-Reversibility: Applications to Partial Differential Equations. Elsevier, New York (1969)

23. Eldén, L: Approximations for a Cauchy problem for the heat equation. Inverse Probl. 3(2), 263-273 (1987)

24. Qian, Z, Fu, CL, Xiong, XT: A modified method for a nonstandard inverse heart conduction problem. Appl. Math. Comput. 180(2), 453-468 (2006)

25. Dang, DT, Nguyen, NI: Regularization and error estimates for nonhomogeneous backward heat problems. Electron. J. Differ. Equ. 2006, 4 (2006)

26. Klibanov, MV, Santosa, F: A computational quasi-reversibility method for Cauchy problems for Laplace's equation SIAM J. Appl. Math. 51(6), 1653-1675 (1991)

27. Qian, Z, Fu, CL, Xiong, XT: Fourth-order modified method for the Cauchy problem for the Laplace equation. J. Comput. Appl. Math. 192(2), 205-218 (2006)

28. Qin, HH, Wei, T: Quasi-reversibility and truncation methods to solve a Cauchy problem for the modified Helmholtz equation. Math. Comput. Simul. 80, 352-366 (2009)

29. Abramowitz, M, Stegun, IA: Handbook of Mathematical Functions. Dover, New York (1972)

30. Engl, HW, Hanke, M, Neubauer, A: Regularization of Inverse Problems. Mathematics and Its Applications, vol. 375. Kluwer Academic, Dordrecht (1996) 